\title{
Montmorillonite-based polyacrylamide hydrogel rings for controlled vaginal drug delivery
}

\begin{abstract}
Vaginal drug delivery is regarded as a promising route against women-related health issues such as unwanted pregnancies and sexually transmitted infections. However, only a very few studies have been reported on the use of hydrogel rings with low cytotoxicity for vaginal drug delivery applications. Moreover, the effect of nanoparticles on hydrogel vaginal rings has not been clearly evaluated. To overcome these challenges, we hereby developed nanocomposite hydrogel rings based on polyacrylamide-sodium carboxymethyl cellulose-montmorillonite nanoparticles in the ring-shaped aluminum mold for controlled drug delivery. The hydrogel rings were synthesized by using $\mathrm{N}, \mathrm{N}^{\prime}$-methylene bisacrylamide, $\mathrm{N}, \mathrm{N}, \mathrm{N}^{\prime}, \mathrm{N}^{\prime}$-tetramethyl ethylene diamine, and ammonium persulfate, as a crosslinker, accelerator, and initiator, respectively. The obtained rings were $5.5 \mathrm{~cm}$ in diameters and $0.5 \mathrm{~cm}$ in rims. Chemical structures of the nanocomposite rings were confirmed by Fourier transform infrared, and Nuclear Magnetic Resonance spectroscopies. Additionally, the swelling ratio of hydrogels was appeared to be adjusted by the introduction of nanoparticles. In vitro release experiment of methylene blue, as a hydrophilic model drug, revealed that the nanocomposite rings could not only reduce burst effect (almost more than twice), but also achieve prolonged release for 15 days in the vaginal fluid simulant which mimic the vaginal conditions at $\mathrm{pH}$ of almost 4.2, and a temperature of $37{ }^{\circ} \mathrm{C}$. Importantly, the resultant hydrogel rings with or without various concentrations of montmorillonite showed low cytotoxicity toward human skin fibroblasts. Furthermore, different antibacterial activities against Escherichia coli were observed for various concentrations of montmorillonite in hydrogels. These results suggest the great potential of montmorillonite-based hydrogel rings for vaginal drug delivery.
\end{abstract}

Keyword: Nanocomposite hydrogel rings; Vaginal drug delivery; Montmorillonite nanoparticles, controlled release; Acrylamide 